\title{
SOME USES OF THE SECOND CONFORMAL STRUCTURE ON STRICTLY CONVEX SURFACES
}

\author{
TILLA KLOTZ
}

1. An oriented surface $S$ immersed smoothly in $E^{3}$ has a conformal structure imposed upon it by the metric of the surrounding space. Thus $S$ may be viewed as a Riemann surface $R_{1}$. But if $S$ is strictly convex (and oriented so that mean curvature $H>0$ ) then the second fundamental form is positive definite and determines still another conformal structure on $S$. Thus $S$ may be viewed as a second Riemann surface $R_{2}$.

In [4], Heinz Hopf uses the $R_{1}$ structure on a surface of constant mean curvature as a basis for one of several proofs that a closed surface of genus zero with constant $H$ must be a sphere. In passing, he derives a convenient formula for the index of an isolated umbilic on an arbitrary surface. This formula involves second derivatives of the functions describing the immersion in $E^{3}$ of the surface in question.

We imitate Hopf's procedures in this paper, restricting our attention to strictly convex surfaces and using $R_{2}$ in place of $R_{1}$ structure. We show that Gauss curvature $K>0$ plays a role relative to $R_{2}$ structure which $H$ plays relative to $R_{1}$ structure. As a consequence we obtain a new proof of Liebmann's theorem that a closed surface with constant $K$ must be a sphere. In addition we too get a formula for the index of an isolated umbilic (at which $K>0$ ). But our formula involves only first derivatives of the functions describing the immersion of the surface in $E^{3}$. The paper closes with a brief comment on Carathéodory's conjecture.

2. We begin with a sketch of the material (pp. 79-86 of [4]) which serves as a model for our own procedures. Isothermal coordinates $x, y$ are introduced locally on $S$ yielding the conformal parameter $z=x+i y$ on $R_{1}$. As a result,

while

$$
\begin{aligned}
I & =\lambda\left(d x^{2}+d y^{2}\right), \\
I I & =L d x^{2}+2 M d x d y+N d y^{2}
\end{aligned}
$$

$$
K=\frac{L N-M^{2}}{\lambda^{2}}, \quad B=\frac{L+N}{2 \lambda} .
$$

Presented to the Society, April 13, 1962; received by the editors April 16, 1962. 
The directions of principal curvature depend only upon $I I$, and are given by

$$
-M d x^{2}+(L-N) d x d y+M d y^{2}=0
$$

If we set

$$
\phi=\frac{L-N}{2}-i M
$$

then (1) becomes

$$
\operatorname{Im}\left\{\phi d z^{2}\right\}=0 .
$$

But this means that the tangent element $d z$ of a line of curvature satisfies

$$
\arg d z=\frac{m \pi}{2}-\frac{1}{2} \arg \phi
$$

where $m$ is an integer.

Umbilics on $S$ correspond to zeros of $\phi$, and thus the index $j$ of an isolated umbilic $U$ at $z=0$ is given by

$$
=\frac{-1}{2 \pi}\left(\frac{1}{2} \Delta \arg \phi\right),
$$

where the change in argument is taken (here and everywhere) as $z$ traverses a sufficiently small circle $|z|=\epsilon$ once in the positive sense. However, if $X$ describes the immersion of $S$ in $E^{3}$, then

$$
\phi=-2 X_{z} \cdot N_{z},
$$

where

$$
\frac{\partial}{\partial z}=\frac{1}{2}\left(\frac{\partial}{\partial x}-i \frac{\partial}{\partial y}\right), \quad \frac{\partial}{\partial \bar{z}}=\frac{1}{2}\left(\frac{\partial}{\partial x}+i \frac{\partial}{\partial y}\right),
$$

and where $N$ is the unit normal to $S$. It follows that

$$
\Omega=\phi d z^{2}
$$

is invariant under changes of isothermal coordinates on $S$, making $\Omega$ a (not necessarily holomorphic) quadratic differential on $R_{1}$. Moreover,

$$
=\frac{-n}{2}
$$

where 


$$
n=\frac{1}{2 \pi} \Delta \arg \phi=\frac{1}{2 \pi} \Delta \arg X_{z} \cdot N_{z} .
$$

The role of $H$ on $R_{1}$ is indicated by writing the Codazzi equations in the form

$$
\phi_{\bar{z}}=\lambda H_{z} \text {. }
$$

Thus $H$ is a constant on $S$ if and only if $\phi$ is an analytic function of $z$, that is if and only if $\Omega$ is a holomorphic quadratic differential on $R_{1}$. (As a result, any $S$ with constant $H$ has isothermal lines of curvature coordinates in the neighborhood of any point which is not an umbilic, which means that any such $S$ is isothermal.) Moreover, since a holomorphic quadratic differential on closed Riemann surface of genus zero must vanish identically, it follows that a closed surface $S$ of genus zero with $H$ constant is composed entirely of umbilics, and must therefore be a sphere.

3. Consider a surface $S$ which is $C^{4}$ immersed in $E^{3}$, for which $K>0$, and which is oriented so that $H>0$. Then $C^{3}$ bisothermal coordinates $u, v$ may be introduced locally on $S$ (see $\$ 4$ of [1], for example), yielding the conformal parameter $w=u+i v$ on $R_{2}$. In terms of bisothermal coordinates,

$$
\begin{aligned}
I & =E d u^{2}+2 F d u d v+G d v^{2}, \\
I I & =\mu\left(d u^{2}+d v^{2}\right),
\end{aligned}
$$

while

$$
K=\frac{\mu^{2}}{W}, \quad H=\frac{\mu(E+G)}{2 W},
$$

where

$$
W=E G-F^{2} \text {. }
$$

The directions of principal curvature depend only on $I$, and are given by

$$
-F d u^{2}+(E-G) d u d v+F d v^{2}=0
$$

If we set

$$
\hat{\phi}=\frac{E-G}{2}-i F
$$

then (3) becomes

$$
\operatorname{Im}\left\{\hat{\phi} d w^{2}\right\}=0
$$


Zeros of $\hat{\phi}$ correspond to umbilics on $S$, while since

$$
\hat{\phi}=2 X_{w} \cdot X_{w},
$$

it is an easy matter to check that

$$
\hat{\Omega}=\hat{\phi} d w^{2}
$$

is invariant under changes of bisothermal coordinates on $S$, making $\hat{\Omega}$ a (not necessarily holomorphic) quadratic differential on $R_{2}$.

We will use the Gauss equations in the form

$$
\begin{aligned}
& X_{u u}=\Gamma_{11}^{1} X_{u}+\Gamma_{11}^{2} X_{v}+\mu N, \\
& X_{u v}=\Gamma_{12}^{1} X_{u}+\Gamma_{12}^{2} X_{v}, \\
& X_{v v}=\Gamma_{22}^{1} X_{u}+\Gamma_{22}^{2} X_{v}+\mu N,
\end{aligned}
$$

where

$$
\begin{array}{ll}
\Gamma_{11}^{1}=\frac{G E_{u}-2 F F_{u}+F E_{v}}{2 W}, & \Gamma_{11}^{2}=\frac{2 E F_{u}-E E_{v}-F E_{u}}{2 W}, \\
\Gamma_{12}^{1}=\frac{G E_{v}-F G_{u}}{2 W}, & \Gamma_{12}^{2}=\frac{E G_{u}-F E_{v}}{2 W}, \\
\Gamma_{22}^{1}=\frac{2 G F_{v}-G G_{u}-F G_{v}}{2 W}, & \Gamma_{22}^{2}=\frac{E G_{v}-2 F F_{v}+F G_{u}}{2 W},
\end{array}
$$

so that

$$
\begin{aligned}
& \Gamma_{11}^{1}+\Gamma_{22}^{2}=\frac{W_{u}}{2 W}, \\
& \Gamma_{22}^{2}+\Gamma_{12}^{1}=\frac{W_{v}}{2 W} .
\end{aligned}
$$

Note that on $R_{\mathbf{2}}$ the Codazzi equations become

$$
\begin{aligned}
& \mu_{u}=\mu\left(\Gamma_{12}^{2}-\Gamma_{22}^{1}\right), \\
& \mu_{v}=\mu\left(\Gamma_{12}^{1}-\Gamma_{11}^{2}\right) .
\end{aligned}
$$

Lemma. $K>0$ is a constant on $S$ if and only if $\hat{\Omega}$ is a holomorphic quadratic differential on $R_{\mathbf{2}}$.

Proof of Lemma. The quadratic differential $\hat{\Omega}$ is holomorphic on $R_{2}$ if and only if $\hat{\phi}$ is analytic in $w$, that is, if and only if

$$
X_{w} \cdot X_{w \bar{w}}=0 \text {. }
$$


But (8) is equivalent to

$$
\left(X_{u u}+X_{v v}\right) \cdot X_{u}=\left(X_{u u}+X_{v v}\right) \cdot X_{v}=0,
$$

which, in view of (6), is equivalent to

$$
\Gamma_{11}^{1}+\Gamma_{22}^{1}=\Gamma_{11}^{2}+\Gamma_{22}^{2}=0 .
$$

On the other hand, $K$ is a constant if and only if $K_{u}=K_{v}=0$, so that

$$
2 \mu_{u} W-\mu W_{u}=2 \mu_{v} W-\mu W_{v}=0 .
$$

We may rewrite (10), using (7), to read

$$
\begin{aligned}
& \mu_{u}=\mu\left(\Gamma_{11}^{1}+\Gamma_{12}^{2}\right), \\
& \mu_{v}=\mu\left(\Gamma_{22}^{2}+\Gamma_{12}^{1}\right) .
\end{aligned}
$$

But then the Codazzi equations force (9) to hold, so that $K$ is a constant if and only if $\hat{\Omega}$ is holomorphic on $R_{2}$.

CoRollary. If $K>0$ is constant on $S$ then $S$ is bisothermal, that is there exist bisothermal lines of curvature coordinates in the neighborhood of any point on $S$ which is not an umbilic.

Proof of Corollary. In the neighborhood of any point which is not a zero of the holomorphic quadratic differential $\hat{\Omega}$, a conformal parameter $w=u+i v$ on $R_{2}$ may be chosen in terms of which $\hat{\phi} \equiv 1$ (see $\S 8$, A of [1]). But then $F=0$ for the bisothermal coordinates $u, v$ on $S$, which are therefore lines of curvature coordinates as well. One can picture the situation by noting that the trajectories and orthogonal trajectories of $\hat{\Omega}$ on $R_{2}$ form a net of lines of curvature on $S$.

It is now an easy matter to give a new proof of Liebmann's theorem. For, if $S$ is a closed surface with constant $K$, we know that $K>0$ and that $S$ has genus zero. Applying the Lemma, we see that $\hat{\Omega}$ is holomorphic on a closed Riemann surface $R_{2}$ of genus zero, so that $\hat{\Omega} \equiv 0$, all points of $S$ are umbilic, and $S$ must be a sphere.

4. We now use (4) to compute the index $j$ of an isolated umbilic $U$ on an arbitrary surface $S$. As noted in [3], if $K=0$ at $U, S$ may be reflected in a suitable sphere, preserving lines of curvature and the index $j$ of $U$, but making $K>0$ in a neighborhood of $U$. We therefore assume that $K>0$ at $U$, and introduce bisothermal coordinates $u, v$ in a neighborhood of $U$.

From (4) it follows that the tangent element $d w$ of a line of curvature satisfies 


$$
\arg \hat{\phi}+2 \arg (d w)=m \pi,
$$

where $m$ is an integer. Thus

$$
\arg d w=\frac{m \pi}{2}-\frac{1}{2} \arg \hat{\phi},
$$

and the index

$$
j=\frac{1}{2 \pi} \Delta \arg d w
$$

of $U$ is given by

$$
j=\frac{-1}{2 \pi}\left(\frac{1}{2} \Delta \arg \hat{\phi}\right) .
$$

But then (5) implies that

$$
j=\frac{-n}{2},
$$

where

$$
n=\frac{1}{2 \pi} \Delta \arg \hat{\phi}=\frac{1}{2 \pi} \Delta \arg X_{w} \cdot X_{w} .
$$

Note that $j$ depends solely upon first derivatives of $X$ (although those must be taken with respect to bisothermal coordinates on a strictly convex $S$ ).

5. Carathéodory's conjecture that there exist at least two umbilics on any smooth ovaloid (really on any smooth closed surface of genus zero in $E^{3}$ ) has been proved only for analytic surfaces. On a closed surface of genus zero with a finite number of umbilics, the sum of the indices of the umbilics must be 2 . Thus any closed genus zero surface has one umbilic $U$, and if

$$
j \leqq 1
$$

for $U$, a second umbilic must exist on the surface. In [2], [3] and [5], it is shown that (11) holds so long as the surface is analytic near $U$. It remains to verify (11) in the nonanalytic case.

We note the elementary fact that (11) amounts to the statement that

$$
n=\frac{1}{2 \pi} \Delta \arg X_{w} \cdot X_{w} \geqq-2,
$$


where we make the surface strictly convex near $U$ if necessary, and use bisothermal coordinates. Clearly, if $K$ is constant near $U$, our Lemma implies that $n \geqq 0$, and (11) must hold. The corresponding remark can be made using Hopf's formula (2) for $j$ in case $H$ is constant near $U$. But then $S$ is analytic near $U$ because of Bernstein's theorem and no new information is obtained (see p. 108 of [4]).

\section{REFERENCES}

1. Lipman Bers, Quasiconformal mappings and Teichmüller's theorem, Conference on Analytic Functions, pp. 89-119, Princeton Univ. Press, Princeton, N. J., 1960.

2. G. Bol, Über Nabelpunkte auf Einer Eifläche, Math. Z. 49 (1943-44), 389-410.

3. H. Hamburger, Beweis Einer Caratheodoryschen Vermutung. I, Ann. of Math. (2) 41 (1940), 63-86; II, III, Acta Math. 73 (1941), 174-332.

4. Heinz Hopf, Lectures on differential geometry in the large, Mimeographed Notes, Stanford University, 1956.

5. Tilla Klotz, On G. Bol's proof of Caratheodory's conjecture, Comm. Pure Appl. Math. 12 (1959), 277-311.

University of California, Los Angeles 